\title{
ALTERAÇÕES NOS ATRIBUTOS QUÍMICOS DE UM LATOSSOLO DISTROFÉRRICO DECORRENTES DA GRANULOMETRIA E DOSES DE CALCÁRIO EM SISTEMAS PLANTIO DIRETO E CONVENCIONAL ${ }^{(1)}$
}

\author{
J . C. A. MELLO(2), R. L. VILLAS BÔAS(3), \\ E. V. LIMA(4), C. A. C. CRUSCIOL ${ }^{(5)} \&$ L. T. BÜLL ${ }^{(6)}$
}

\begin{abstract}
RESUMO
Atualmente, percebe-se o interesse na calagem superficial, sem prévia incorporação, para instalação do sistema plantio direto (SPD). Dessa forma, objetivou-se determi nar os efeitos de granulometria e doses de calcários no SPD, em fase de implantação, e no sistema de plantio convencional (SPC) sobre o pH, $\mathrm{H}+\mathrm{Al}, \mathrm{Ca}^{2+} \mathrm{e} \mathrm{Mg}^{2+}$. O experimento foi realizado no ano agrícola de 1998/99, na FCA/U NESP-Botucatu (SP), em Latossolo Vermelho. O delineamento experimental foi de blocos ao acaso, com parcelas subsubdivididas e quatro repetições. As parcelas representaram os sistemas de plantio (SPD e SPC); as subparcelas, a granulometria dos calcários [grosso (PRNT $=56 \%$ ) e fino (PR NT $=90 \%$ )], e as subsubparcelas, as doses de 2, 4 e $6 \mathrm{t} \mathrm{ha-1}$ (calcário grosso) e 1,2; 2,4 e 3,6 t ha-1 (fino). O solo foi amostrado, a 0-5, 5-10, 10-20 e 20-40 cm de profundidade, 1, 3 e 12 meses após a aplicação dos corretivos. A análise de variância não detectou interação tripla entre os fatores. A aplicação de calcário superficial no SPD, independentemente da granulometria e da dose, alterou positivamente os atributos químicos do solo $(0-5$ e 5-10 cm), 12 meses após a calagem. O corretivo continuou reagindo, independentemente do sistema de plantio, de forma intensa, mesmo após três meses. A aplicação de doses mais elevadas de calcário, com maior granulometria, sugeriu efeito residual prolongado.
\end{abstract}

Termos de indexação: plantio direto, calagem, acidez, fertilidade do solo, mobilidade de cátions.

\footnotetext{
(1) Parte da Tese de Mestrado apresentada pelo primeiro autor à Faculdade de Ciências Agronômicas da Universidade Estadual Paulista - FCA/UNESP. Recebido para publicação em junho de 2002 e aprovado em março de 2003.

(2) Engenheiro-Agrônomo, MSc, Empresa Brasileira de Calcário Ltda. - EMBRACAL. Rodovia SP-127, Km 56, Caixa Postal 41, CEP 13440-000 Saltinho (SP). E-mail: julioassad@bol.com.br

(3) Professor Adjunto do Departamento de Recursos Naturais, Facul dade de Ciências Agronômicas da UniversidadeEstadual Paulista - FCA/UNESP. Caixa Postal 237, CEP 18603-970 Botucatu (SP).

(4) Engenheiro-Agrônomo, MSc, Universidade Federal Rural da Amazônia - UFRA. Av. Pres. Tancredo Neves s/n, Caixa Postal 917, CEP 66077-530 Belém (PA). Bolsista CAPES/PICDT/FCAP. E-mail: valle@fca.unesp.br

(5) Professor Adjunto do Departamento de Produção Vegetal, FCA/UNESP. Bolsista CNPq. E-mail: crusciol @fca.unesp.br

(6) Professor Titular do Departamento de Recursos Naturais, FCA/UNESP. E-mail: bull@fca.unesp.br
} 


\title{
SUMMARY: CHANGES OF CHEMICAL SOIL CHARACTERISTICS DUE TO DOSES AND PARTICLE SIZES OF LIMESTONE IN NO- TILLAGE AND CONVENTIONAL SYSTEMS
}

\begin{abstract}
Theinterest of surfaceliming of soils on no-tillage system is increasing. The objective of this work was to evaluate the effects of different lime particle sizes and doses applied during the implantation phase of no-tillage and in conventional tillage systems on soil pH and $\mathrm{H}+\mathrm{Al}, \mathrm{Ca}^{2+}$, and $\mathrm{Mg}^{2+}$ contents. The experiment was carried out in 1998/ 99, on the experimental farm of São Paul o StateU niversity in Botucatu, São Paulo, Brazil, on a Red Distroferric Latossol (Oxisol). A split plot experimental design with four replications was used. Thenumber of plots represented thetillagesystem, thesub-plots represented particle sizes of limestone (large and small) and sub-sub plots lime doses of 2; 4 and $6 \mathrm{t} \mathrm{ha}^{-1}$ (large limestone particles) and 1.2; 2.4 and $3.6 \mathrm{t} \mathrm{ha}^{-1}$ (small limestone particles). The soil was sampled (at depths of $0-5,5-10,10-20$ and $20-40 \mathrm{~cm}$ ) 1, 3 and 12 months after liming. Variance analysis did not detect a threefold interaction among the factors. 12 months after surfaceliming, chemical soil characteristics werepositively affected $(0-5$ and $5-10 \mathrm{~cm})$ in theno-tillage system, independent of particlesizeand doses. Even after morethan three months, thefertilizer had a conti nuos strong effect, independent of thetill agesystem. Higher lime doses with large particle sizes provided a prolonged residual effect.
\end{abstract}

Index terms: no-tillage, liming, acidity, soil fertility, cation mobility.

\section{NTRODUÇÃO}

A área estimada sob sistema plantio direto(SPD) no planeta representa $0,4 \%$ do total cultivado, o que, em valor numérico, significa 55 milhões de ha, estando $82 \%$ no continente americano (Derpsch \& Florentin, 2000). No Brasil, estima-se que a área ocupada com SPD éde aproximadamente 13 mil hões de ha, o que representa quase $24 \%$ do total no mundo.

O SPD pode ser dividido em duas fases: a de instalação, correspondente aos primeir ros quatro ou cinco anos após o início do sistema, e a de estabilidade, em que claramente são observadas alterações nas propriedades químicas, físicas e biológicas do solo (Salet, 1994).

A calagem, em áreas onde o SPD já se encontra estabel ecido, érealizada na superfície do solo (Caires et al., 1998; 1999). I sso tem gerado intenso questionamento quanto à eficiência ou não dessa prática (Sá, 1995), pois os corretivos da acidez, à base de carbonatos de cálcio e magnésio, são pouco solúveis em água, tendo os produtos de sua reação com o solo mobilidade limitada no perfil (Caires et al., 1998). No entanto, as evidências quanto à ação benéfica da calagem superficial, atuando nas primeiras camadas abaixo da superfície do sol o (0-5 e 5-10 cm), vêm sendo observadas em diferentes condições de solo e clima. Assim, dentre tantos trabalhos realizados, os de Caires et al. (1998) e Pöttker \& Ben (1998) confirmaram que o cal cário em superfície corrige a acidez, aumentando significativamente o $\mathrm{pH}$ e el evando os teores de $\mathrm{Ca}$ e Mg trocáveis do sol o atéà profundidade de $5 \mathrm{~cm}$ e, em menor grau, na camada de $5-10 \mathrm{~cm}$.
No sistema de plantio convencional (SPC) ou na fase de instalação do SPD, a correção da aci dez pressupõe a incorporação do calcário ao solo com aração e gradagens (Rheinheimer et al., 2000), para proporcionar o máximo contato entre as partículas do corretivo com os colói des do sol o (Sá, 1995). Entretanto, percebe-se, atualmente, grande interesse na busca de formas alternativas para estabel ecimento do SPD, sem incorporação prévia do cal cário, não havendo a necessidade de promover o revolvimento inicial do solo por meio de preparo convencional, real izando-se a cal agem superficial desde o início (Caires et al., 2000; Delavale et al., 2000; Lima \& Crusciol, 2001). As vantagens desse procedimento, segundo Caires et al. (2000), estariam relacionadas com a manutenção dos atributos químicos eda estrutura do solo, com o maior controle da erosão ecom a economia com as operações de incorporação de calcário e preparo do solo.

No SPD, o cal cário apresenta reação mais lenta e maior poder residual, o que pode estar associado à não-movimentação do sol o eà menor mineralização da matéria orgânica, quando comparado ao SPC (Souza, 2000). Por esse motivo, levanta-se a hipótese de que cal cários mais reativos, ou seja, aqueles que se apresentam mais finos ou com um grau de moagem mai or, corrigiriam, de forma mais eficiente, a acidez dos sol os em SPD. O tamanho das partículas condiciona a taxa de reatividade do calcário, uma vez que a velocidade de neutralização depende da área superficial do corretivo em contato com o solo. Assim, quanto mais fino o corretivo, mais rápida a reação do material, desde que o solo esteja úmido (Alcarde et al., 1989; Tedesco \& Gianello, 1989).

No Brasil, a capacidade neutralizante dos corretivos é conhecida como poder relativo de 
neutralização total (PRNT), que considera a pureza química da rocha de origem, denominada poder de neutralização (PN), e o grau de moagem, chamado de taxa de reatividade (RE), ambos expressos em percentagem de equivalência ao $\mathrm{CaCO}_{3}$ puro (Quaggio, 2000). Assim, quanto maior a pureza química da rocha calcária e sua $\mathrm{RE}$, maior será o PRNT e menor deverá ser o efeito residual do cal cário aplicado no solo. Ressalta-se que o val or de RE indica a capacidade neutralizante no curto período de três meses, como prevê a legislação brasileira atual, de acordo com trabal ho de Alcarde et al. (1989). No médio e longo prazo, a capacidade neutralizante deverá ser maior, condicionada por uma série de fatores ligados ao sistema de produção adotado e ao ambiente.

O tempo de reação do calcário aplicado na superfície do solo em SPD também pode variar de acordo com a dose utilizada. Trabal ho como o de Pöttker \& Ben (1998) indica que a necessidade de calcário no SPD talvez seja menor do que no SPC. Nesse contexto, merecem atenção especial as altas produtividades obtidas em SPD, na ausência de calcário, em solos com elevada acidez, como as obtidas por Caires et al. (1998). I sso evidencia que há outros mecanismos envolvidos na tolerância de plantas ao $\mathrm{Al}^{3+}$ e na absorção de nutrientes. Daí surge o interesse em avaliar, além da dose total de cal cário recomendada para oSPC, segundo a análise de solo na profundidade de $0-20 \mathrm{~cm}$, frações dessa dose, com o intuito de verificar se a sua redução em SPD acarretaria alterações significativas nos atributos químicos do solo.

O presente trabal ho teve por objetivo avaliar os efeitos de granulometria e doses de cal cários no plantio direto, em fase de estabelecimento, e convencional sobre alguns atributos químicos do perfil do solo.

\section{MATERIAL E MÉTODOS}

O experimento foi realizado durante o ano agrícola de 1998/99, na Fazenda Experimental Lageado, pertencente à Faculdade de Ciências
Agronômicas/UNESP, no município de Botucatu (SP), a latitude $22^{\circ} 58^{\prime} \mathrm{S}$, longitude $48^{\circ} 23^{\prime} \mathrm{W}$ e altitude de $775 \mathrm{~m}$. A precipitação pluvial total duranteotranscorrer do experimentofoi de $1.943 \mathrm{~mm}$. O solo da área experimental é classificado como Latossolo Vermel ho distroférrico(EMBRAPA, 1999).

A composição granulométrica do solo, avaliada na camada de $0-20 \mathrm{~cm}$ de profundidade, revelou $50 \mathrm{~g} \mathrm{~kg}^{-1}$ de areia no solo, $360 \mathrm{~g} \mathrm{~kg}^{-1}$ de silte no sol o e $590 \mathrm{~g} \mathrm{~kg}^{-1}$ de argila no sol o. Os atri butos químicos do solo, antes da instalação do experimento, estão apresentados em quatro profundidades, 0-5, 5-10, 10-20 e 20-40 cm (Quadro 1) e o método utilizado na análise encontra-se descrito em Raij \& Quaggio (1983).

O delineamento experimental adotado foi de blocos ao acaso, com parcelas subsubdivididas e quatro repetições. As parcelas representaram os sistemas de plantio (SPD e SPC), cujas dimensões foram de 5,4 m delargura por 36 m de comprimento, com área total de $194,4 \mathrm{~m}^{2}$. As subparcelas representaram as granulometrias do cal cário grosso (PRNT $=56 \%$ ) e fino (PRNT $=90 \%$ ), tendo, por conseguinte, área de 97,2 $\mathrm{m}^{2}$. As subsubparcel as de 32,4 $\mathrm{m}^{2}$ representaram as doses de calcário: 2; 4 e $6 \mathrm{t} \mathrm{ha}^{-1}$, para o material grosso, e de 1,$2 ; 2,4 \mathrm{e}$ $3,6 \mathrm{t} \mathrm{ha}^{-1}$, para o material fino, equivalentes a $1 / 3$, $2 / 3$ e $3 / 3$ da dose para el evar a $V \%=70$, conforme recomendação de Raij \& Cantarella (1997), para a cultura do milho. As doses foram calculadas levandose em consi deração as médias aritméticas de CTC e V \% das camadas de 0-5, 5-10 e 10-20 cm (Quadro 1).

Os cal cários utilizados foram dolomíticos, de origem sedimentar, provenientes do município de I peúna (SP). Para o calcário fino, utilizaram-se apenas partículas passantes na peneira n०50 ( $<0,30 \mathrm{~mm}$ ), o que conferiu uma RE de $100 \%$. As análises (química efísica) dos cal cários empregados no experimento encontram-se no quadro 2 , segundo os procedimentos descritos pelo Lanarv (1988).

A área experimental, por vários anos, foi manejada de modo convencional, constando de arações e gradagens. Nos três anos anteriores à instalação do trabalho (1995, 96 e 97), o local encontrava-se sob pousio, sendo a cobertura vegetal

Quadro 1. Atributos químicos do solo antes da instalação do experimento

\begin{tabular}{|c|c|c|c|c|c|c|c|c|c|c|}
\hline Profundidade & $\mathrm{pH}\left(\mathrm{CaCl}_{2}\right)$ & MO(1) & P (resina) & $\mathbf{H}+\mathbf{A l}$ & $\mathbf{K}^{+}$ & $\mathrm{Ca}^{2+}$ & $\mathbf{M g}^{2+}$ & $\mathbf{S B}^{(2)}$ & $\mathbf{T}^{(3)}$ & $V^{(4)}$ \\
\hline $\mathrm{cm}$ & & $\mathrm{g} \mathrm{dm}^{-3}$ & $\mathrm{mg} \mathrm{dm}-3$ & 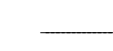 & 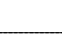 & $-\mathrm{mmol}$ & $\mathrm{m}^{-3}$ & 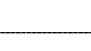 & - & $\%$ \\
\hline $0-5$ & 4,4 & 31 & 6 & 58 & 2,8 & 14 & 9 & 26 & 84 & 31 \\
\hline $5-10$ & 4,5 & 33 & 5 & 61 & 4,0 & 14 & 10 & 28 & 89 & 32 \\
\hline $10-20$ & 4,5 & 34 & 3 & 58 & 3,2 & 17 & 11 & 31 & 89 & 35 \\
\hline $20-40$ & 4,4 & 23 & 4 & 68 & 2,8 & 14 & 8 & 25 & 92 & 27 \\
\hline
\end{tabular}

(1) $\mathrm{MO}=$ matéria orgânica. ${ }^{(2)} \mathrm{SB}=$ soma de bases. ${ }^{(3)} \mathrm{T}=$ capacidade de troca de cátions a pH 7,0. ${ }^{(4)} \mathrm{V}=$ saturação por bases. 
Quadro 2. Análise química e física dos corretivos de acidez do solo

\begin{tabular}{|c|c|c|}
\hline Atributo & Calcário grosso & Calcário fino \\
\hline Umidade (\%) & 5,00 & 0,57 \\
\hline $\mathrm{CaO}(\%)$ & 20,50 & 27,50 \\
\hline $\mathrm{MgO}(\%)$ & 13,60 & 18,50 \\
\hline Retido na Peneira $\mathrm{n}^{\circ} 10$ (> que $2 \mathrm{~mm}$ ) & 0,40 & 0,0 \\
\hline Retido na Peneira n²0 (até 0,84 mm) & 10,69 & 0,0 \\
\hline Retido na Peneira $\mathrm{n}^{\circ} 50$ (> que $0,30 \mathrm{~mm}$ ) & 29,36 & 0,0 \\
\hline Poder de Neutralização (PN) & 70,50 & 95,00 \\
\hline Reatividade (RE) & 78,94 & 100,00 \\
\hline PRNT & 56,00 & 95,00 \\
\hline
\end{tabular}

composta por gramíneas, predominantemente Brachiaria decumbens. A aplicação do material corretivo foi efetuada manualmente no dia 30/11/ 98, sendo realizada, imediatamente, a incorporação do calcário nas parcelas que tinham como tratamento oSPC. Foi utilizada uma gradearadora, que trabalhou à profundidade média de $20 \mathrm{~cm}$, seguida de uma grade niveladora, que atingiu $15 \mathrm{~cm}$ de profundidade em média. Para oSPD, os cal cários foram aplicados sobre uma cobertura vegetal morta deaveia preta (Avena strigosa), que, também, estava presente nas parcelas do SPC.

O cultivo do milho foi feito no período de 23/12/ 98 a 28/05/99, com uma população média de 55.000 plantas ha-1. Como adubação de semeadura, empregaram-se $320 \mathrm{~kg} \mathrm{ha}^{-1}$ da fórmula 8-28-16, de acordo com a recomendação de Raij \& Cantarella (1997), baseando-se na média dos dados fornecidos pelo quadro 1 até à profundidade de $20 \mathrm{~cm}$. A adubação de cobertura foi efetuada aos 30 dias da emergência, utilizando-se $55 \mathrm{~kg} \mathrm{ha}^{-1}$ de $\mathrm{N}$ na forma de uréia (Raij \& Cantarella, 1997). Após a colheita, rol ou-se o resto cultural com rolo-faca, como forma de proteçãosuperficial dosoloatéofinal do experimento.

As amostragens desolo foram real izadas nos dias: 4/01/99 (1 a amostragem), aproximadamente 1 mês após a aplicação dos corretivos; 3/03/99 (2a amostragem), aproximadamente três meses após a aplicação, e 13/12/99 (3a amostragem), aproximadamente 12 meses após a aplicação do corretivo.

As profundidades amostradas foram de 0-5, 510, 10-20 e 20-40 cm, retirando-se cinco amostras simples por camada de solo, em cada subsubparcela, realizando-se caminhamento de amostragem na diagonal, tomando-se por referência as entrelinhas de cultivo para pontos de coleta, com a utilização de trado holandês. Antes da transferência da terra coletada (amostras simples) para o balde, com o auxílio de uma faca, excluiu-se o excedentenas duas laterais e foram eliminados, também, 2 a $3 \mathrm{~cm}$ da parte superior einferior, aproveitando apenas o solo do centro do coletor (Raij et al., 1997; Chitolina et al., 1999). As amostras compostas foram secas ao ar $\mathrm{e}$ peneiradas (malha de $2 \mathrm{~mm}$ ), para determinação do $\mathrm{pH}\left(\mathrm{CaCl}_{2}\right)$, acidez potencial $(\mathrm{H}+\mathrm{Al})$, Ca eMgtrocáveis, segundo método descrito em Raij \& Quaggio (1983).

Os resultados foram submetidos à análise de variância, tendo-se comparado as médias pelo teste de Tukey a $5 \%$.

\section{RESULTADOS E DISCUSSÃO}

A análise de variância dos resultados para os atributos químicos avaliados não mostrou interações triplas entre sistema de plantio, granulometria e doses de calcário. Assim, os efeitos isolados desses fatores são discutidos separadamente ou mediante o desdobramento de interações duplas. Ressalta-se que, para os atributos químicos, em determinadas profundidades, o teste $F$ indicou efeito significativo de um fator isolado ou da interação entre dois fatores, mas, em contrapartida, o teste deTukey não detectou diferenças estatísticas na comparação de médias; por essa razão, optou-se por não discutir esses resultados.

$\mathrm{Na} 1^{\mathrm{a}}$ amostragem (Quadro 3), não se observou contraste significativo entre médias pelo teste de Tukey, em qualquer profundidade. I sso se deve ao fato de não ter havido tempo suficiente para que ocorresse a hidrólise da maior parte do calcário (Alcarde et al., 1989) e sua conseqüente liberação de $\mathrm{Ca}^{2+}, \mathrm{Mg}^{2+}, \mathrm{HCO}_{3}{ }^{-}$e $\mathrm{OH}^{-}$. São esses dois ânions os responsáveis pela el evação do pH do solo. Dessa forma, apenas uma pequena parte das frações granulométricas de menor dimensão dos corretivos reagiu em tempo hábil, não al terando praticamente os atributos apresentados no quadro 1 . Nem mesmo o elevado índice de precipitação pluvial (300 mm), no mês subseqüente à aplicação do calcário (dezembro de 1998), contribuiu para uma rápida reação do corretivo no solo, uma vez que este apresenta, por natureza, baixo grau de sol ubilização, necessitando de mais tempo para expressar seu potencial de neutralização. 
Quadro 3. Valores de pH e de acidez potencial (H + Al) e teores de cálcio trocável do solo em diferentes profundidades, após 1, 3 e 12 meses da aplicação do corretivo, considerando o sistema de plantio (SP), granulometria (Gran) e doses de calcário (Doses)

\begin{tabular}{|c|c|c|c|c|c|c|c|c|c|c|c|c|}
\hline \multirow{2}{*}{ Fator } & \multicolumn{4}{|c|}{ 1’a amostragem (1 mês) - Prof. (cm) } & \multicolumn{4}{|c|}{$2^{\mathrm{a}}$ amostragem (3 meses) - Prof. (cm) } & \multicolumn{4}{|c|}{$3^{a}$ amostragem (12 meses) - Prof. (cm) } \\
\hline & $0-5$ & 5-10 & $10-20$ & $20-40$ & $0-5$ & $5-10$ & $10-20$ & $20-40$ & $0-5$ & $5-10$ & $10-20$ & $20-40$ \\
\hline \multicolumn{13}{|c|}{ pH CaCl 2} \\
\hline \multicolumn{13}{|c|}{ Sistema de plantio } \\
\hline $\begin{array}{l}\text { Convencional } \\
\text { Direto }\end{array}$ & $\begin{array}{l}4,6 a \\
4,6 a\end{array}$ & $\begin{array}{l}4,6 a \\
4,5 a\end{array}$ & $\begin{array}{l}4,5 a \\
4,6 a\end{array}$ & $\begin{array}{l}4,5 a \\
4,5 a\end{array}$ & $\begin{array}{l}4,8 a \\
4,8 a\end{array}$ & $\begin{array}{l}4,9 a \\
4,5 b\end{array}$ & $\begin{array}{l}4,5 \mathrm{a} \\
4,2 \mathrm{~b}\end{array}$ & $\begin{array}{l}4,3 \mathrm{a} \\
4,1 \mathrm{~b}\end{array}$ & $\begin{array}{l}4,9 \mathrm{~b} \\
5,5 \mathrm{a}\end{array}$ & $\begin{array}{l}4,7 a \\
4,7 a\end{array}$ & $\begin{array}{l}4,5 \mathrm{a} \\
4,1 \mathrm{a}\end{array}$ & $\begin{array}{l}3,9 a \\
3,9 a\end{array}$ \\
\hline \multicolumn{13}{|c|}{ Granulometria } \\
\hline Grosso & $4,5 \mathrm{a}$ & $4,5 a$ & $4,6 \mathrm{a}$ & $4,5 a$ & $4,8 \mathrm{a}$ & $4,8 \mathrm{a}$ & $4,4 a$ & $4,2 a$ & $5,2 \mathrm{a}$ & $4,7 a$ & $4,3 \mathrm{a}$ & 3,9 a \\
\hline Fino & 4,6 a & 4,6 a & 4,6 a & $4,5 \mathrm{a}$ & $4,8 \mathrm{a}$ & 4,6 a & $4,4 \mathrm{a}$ & 4,1 a & $5,2 a$ & $4,7 \mathrm{a}$ & $4,3 a$ & $3,9 a$ \\
\hline \multicolumn{13}{|c|}{ Doses } \\
\hline $1 / 3$ & $4,6 a$ & $4,5 a$ & $4,5 \mathrm{a}$ & $4,5 \mathrm{a}$ & $4,9 a$ & $4,8 \mathrm{a}$ & $4,4 \mathrm{a}$ & $4,2 \mathrm{a}$ & $4,9 b$ & $4,6 a$ & $4,3 a$ & $3,9 a$ \\
\hline $2 / 3$ & $4,5 \mathrm{a}$ & $4,5 a$ & $4,5 \mathrm{a}$ & $4,4 a$ & $4,8 \mathrm{a}$ & 4,6 a & $4,3 a$ & $4,2 \mathrm{a}$ & $5,1 \mathrm{ab}$ & $4,6 a$ & $4,3 a$ & $3,9 a$ \\
\hline $3 / 3$ & $4,6 a$ & $4,6 a$ & $4,7 a$ & $4,6 a$ & $4,7 a$ & $4,6 a$ & $4,3 a$ & $4,2 a$ & $5,6 a$ & $4,9 a$ & $4,3 a$ & $3,9 a$ \\
\hline \multicolumn{13}{|c|}{ Valor de F } \\
\hline SP & 0,06 & 6,72 & 0,06 & 0,13 & 0,86 & $12,66^{*}$ & $20,31 *$ & $121,50 *$ & $3,12 *$ & 0,02 & 0,48 & 0,04 \\
\hline Gran & $6,02^{*}$ & 0,21 & 0,30 & 0,02 & 0,10 & 0,36 & 0,02 & 1,90 & 0,14 & 0,62 & 0,22 & 0,59 \\
\hline Dose & 0,06 & 0,84 & 1,73 & 1,44 & 0,71 & 1,22 & 0,65 & 0,26 & $6,02^{*}$ & 2,35 & 0,04 & 0,58 \\
\hline $\mathrm{SP} \times \mathrm{Gran}$ & $6,80^{*}$ & 0,36 & $6,82^{*}$ & 0,84 & 2,18 & 0,25 & 0,58 & 0,12 & $12,97 *$ & $6,02 *$ & 2,81 & 0,92 \\
\hline SP $\times$ Dose & 0,54 & 1,97 & 0,47 & 0,84 & 0,93 & 0,56 & 1,29 & 1,30 & 2,10 & 1,76 & 0,99 & 0,09 \\
\hline Gran x Dose & 2,27 & $3,72^{*}$ & 0,49 & 1,49 & 0,96 & 1,07 & 0,65 & 0,79 & 0,21 & 0,44 & $3,55^{*}$ & 1,35 \\
\hline & & & & & & V. (\%) & & & & & & \\
\hline SP & 2 & 2 & 5 & 4 & 1 & 3 & 3 & 0,5 & 8 & 5 & 5 & 1 \\
\hline Gran & 2 & 5 & 3 & 5 & 7 & 10 & 7 & 3 & 2 & 4 & 3 & 2 \\
\hline Dose & 10 & 7 & 8 & 8 & 4 & 8 & 7 & 4 & 11 & 9 & 5 & 6 \\
\hline & & & & & $\mathbf{H}+\mathbf{A l}$ & nmol & $\left.n^{-3}\right)$ & & & & & \\
\hline & & & & & Siste & a de pla & tio & & & & & \\
\hline Convencional & $62 a$ & $56 \mathrm{~b}$ & $57 a$ & $66 a$ & $56 a$ & $58 \mathrm{~b}$ & $70 \mathrm{~b}$ & $86 \mathrm{~b}$ & $49 a$ & $52 a$ & $62 \mathrm{~b}$ & $87 a$ \\
\hline Direto & $64 a$ & $68 a$ & 64 a & $64 a$ & 58 a & $74 a$ & $92 a$ & $98 a$ & $36 \mathrm{~b}$ & $55 a$ & $82 a$ & $100 \mathrm{a}$ \\
\hline & & & & & Gra & ulometr & & & & & & \\
\hline Grosso & $64 a$ & $62 a$ & $59 a$ & $63 a$ & $57 a$ & $66 a$ & $82 a$ & $92 a$ & $42 a$ & $56 a$ & $74 a$ & $95 a$ \\
\hline Fino & $61 a$ & $61 a$ & $62 a$ & $67 a$ & $57 a$ & $66 a$ & $80 a$ & $93 a$ & $44 a$ & 51 a & $69 a$ & $92 a$ \\
\hline & & & & & & Doses & & & & & & \\
\hline $1 / 3$ & $63 a$ & $62 a$ & $61 a$ & $63 a$ & $55 a$ & $62 a$ & $78 a$ & $93 a$ & $50 a$ & $56 a$ & $73 a$ & $98 a$ \\
\hline $2 / 3$ & $61 a$ & $62 a$ & $62 a$ & $69 a$ & $57 a$ & $67 a$ & $83 a$ & $96 a$ & $44 a$ & $56 a$ & $68 a$ & $88 a$ \\
\hline $3 / 3$ & $65 a$ & $62 a$ & $57 a$ & $62 a$ & $60 a$ & $69 a$ & $83 a$ & $88 a$ & $35 a$ & $45 a$ & $74 a$ & $94 a$ \\
\hline & & & & & & lor de F & & & & & & \\
\hline $\mathrm{SP}$ & 0,19 & $3,30 *$ & 0,32 & 0,03 & 0,19 & $62,15^{*}$ & $252,24 *$ & $1,04^{*}$ & $2,45^{*}$ & 0,38 & 3,83* & 2,47 \\
\hline Gran & 0,45 & 0,01 & 0,15 & 0,13 & 0,02 & 0,01 & 0,06 & 0,20 & 1,81 & 0,98 & 1,27 & 0,03 \\
\hline Dose & 0,12 & 0,01 & 0,54 & 0,74 & 0,57 & 0,75 & 0,37 & 1,13 & 2,82 & 2,84 & 0,65 & 0,53 \\
\hline $\mathrm{SP} \times \mathrm{Gran}$ & 1,72 & 0,12 & 1,48 & 0,30 & 1,45 & 0,53 & 0,31 & 0,60 & 3,97 & 0,04 & 0,26 & 0,03 \\
\hline SP $\times$ Dose & 0,37 & 0,98 & 1,24 & 0,67 & 0,56 & 0,31 & 1,20 & 0,81 & 0,11 & 1,45 & 0,26 & 0,74 \\
\hline Gran x Dose & 0,47 & 2,25 & 0,05 & 1,24 & 0,10 & 0,83 & 2,02 & 0,93 & 0,13 & 1,47 & 0,35 & 0,66 \\
\hline & & & & & & V. (\%) & & & & & & \\
\hline SP & 11 & 16 & 28 & 21 & 8 & 4 & 2 & 20 & 28 & 13 & 20 & 12 \\
\hline Gran & 12 & 23 & 21 & 32 & 22 & 31 & 25 & 15 & 8 & 20 & 12 & 26 \\
\hline Dose & 37 & 25 & 25 & 26 & 22 & 25 & 22 & 18 & 41 & 36 & 22 & 29 \\
\hline & & & & & $\mathrm{Ca}^{2+}$ & imola $_{\text {c }}$ & $-3)$ & & & & & \\
\hline & & & & & Siste & d & & & & & & \\
\hline Convencional & $17 a$ & $17 \mathrm{a}$ & $18 a$ & $16 a$ & 31 a & $32 a$ & $24 a$ & $11 \mathrm{a}$ & $35 b$ & $31 a$ & $26 a$ & $10 a$ \\
\hline Direto & $17 a$ & $14 \mathrm{a}$ & $16 a$ & $21 \mathrm{a}$ & $30 a$ & $18 \mathrm{~b}$ & $10 \mathrm{~b}$ & $8 \mathrm{~b}$ & $48 a$ & $31 \mathrm{a}$ & $16 \mathrm{~b}$ & $8 a$ \\
\hline & & & & & Gro & ulometr & & & & & & \\
\hline Grosso & $15 a$ & $15 a$ & $18 a$ & $22 a$ & $33 a$ & $28 a$ & $20 a$ & $10 a$ & $42 a$ & $30 a$ & $22 a$ & $9 a$ \\
\hline Fino & $19 a$ & $16 a$ & $16 a$ & $15 a$ & $27 a$ & $22 a$ & $14 \mathrm{a}$ & $8 a$ & $42 a$ & $32 a$ & $20 a$ & $9 a$ \\
\hline & & & & & & Doses & & & & & & \\
\hline & $19 a$ & $17 a$ & $15 \mathrm{a}$ & $23 a$ & $32 \mathrm{a}$ & & $17 \mathrm{a}$ & $9 a$ & & $29 a$ & $18 a$ & \\
\hline $2 / 3$ & $15 a$ & $13 a$ & $17 a$ & $15 a$ & $32 a$ & $23 a$ & $15 a$ & $10 \mathrm{a}$ & $40 \mathrm{~b}$ & $28 a$ & $22 a$ & $9 a$ \\
\hline $3 / 3$ & $16 a$ & $18 a$ & $20 a$ & $17 a$ & $28 a$ & $24 a$ & $19 a$ & $8 a$ & $55 a$ & $36 a$ & $22 a$ & $9 a$ \\
\hline & & & & & & Ior de F & & & & & & \\
\hline SP & 0,01 & 3,34 & 0,60 & 0,94 & 0,07 & $15,82^{*}$ & $21,54 *$ & $2,51 *$ & $9,10^{*}$ & 0,01 & $6,33 *$ & $38,62 *$ \\
\hline Gran & $10,61 *$ & 0,09 & 1,77 & 0,58 & 1,36 & 0,85 & 0,96 & 0,82 & 0,00 & 0,23 & 0,17 & 0,11 \\
\hline Dose & 0,80 & 2,39 & 1,21 & 0,95 & 0,44 & 0,67 & 0,18 & 0,57 & $12,68^{*}$ & $2,77 *$ & 1,07 & 0,11 \\
\hline $\mathrm{SP} \times \mathrm{Gran}$ & $10,13^{*}$ & 1,29 & $9,33^{*}$ & 0,13 & 2,85 & 0,30 & 0,85 & 0,18 & 4,94 & 2,14 & 1,20 & 2,59 \\
\hline SP $\times$ Dose & 0,29 & 2,65 & 0,33 & 0,36 & 2,34 & 3,10 & 0,98 & 1,63 & 2,81 & 0,98 & 0,41 & 0,05 \\
\hline Gran x Dose & 2,01 & $3,68^{*}$ & 0,27 & 1,30 & 0,25 & 0,40 & 0,50 & 0,35 & 0,16 & 1,93 & $3,72^{*}$ & 0,57 \\
\hline & & & & & & V. (\%) & & & & & & \\
\hline SP & 17 & & & 42 & & & 24 & & & & & \\
\hline Gran & 14 & 34 & 20 & 84 & 35 & 54 & 65 & 45 & 9 & 27 & 33 & 37 \\
\hline Dose & 65 & 46 & 54 & 94 & 46 & 45 & 89 & 51 & 33 & 36 & 40 & 61 \\
\hline
\end{tabular}

Médias seguidas por letras iguais, nas colunas, não diferem entre si pelo teste de Tukey a 5 \%. * significativo a $5 \%$ pelo teste $\mathrm{F}$. 
Observa-se, na $2^{\mathrm{a}}$ amostragem (Quadro 3), que, de modo geral, houve el evações nos valores de $\mathrm{pH}$, nos sistemas de plantio, a partir da profundidade de $5 \mathrm{~cm}$, decorrentes do mai or tempo de solubilização dos materiais cal cários. Torna-se evidente a mel hor neutralização da acidez nas parcelas nas quais os corretivos foram incorporados (SPC), em relação às parcelas nas quais os corretivos foram aplicados em superfície(SPD). I sso podeser explicado, na medida em que no SPC houve uma maior camada de solo mobilizada mecanicamente(até $20 \mathrm{~cm}$ ), proporcionando a mistura do cal cário com o sol o, o que acel er ou a rápida dissolução do corretivo. J á na camada de 0$5 \mathrm{~cm}$, sobre a qual todo calcário foi depositado no SPD, não foi observada diferença de $\mathrm{pH}$ em relação ao SPC, isto é, após três meses, o corretivo aplicado em superfície reagiu até $5 \mathrm{~cm}$, em virtude de sua elevada concentração, suprindo a necessidade de incorporação no solo.

$\mathrm{Na} 2^{\mathrm{a}}$ e $3^{\mathrm{a}}$ amostragens (Quadro 3), não houve difer enças de comportamento entre os cal cários, fino e grosso, independentemente do sistema de plantio e de doses. Vale lembrar que o PRNT foi levado em consideração para o cál culo da necessidade de calagem, adicionando-se quantidade maior do calcário grosso em relação ao fino, visando elevar, em ambos os casos, a $\vee$ para o mesmo valor. O aumento da dose de cal cário não refletiu, ao contrário do esperado, el evações de $\mathrm{pH}$ na $1^{\mathrm{a}}$ e $2^{\mathrm{a}}$ amostragens.

$\mathrm{O} \mathrm{pH}$ obtido aos 12 meses da aplicação dos corretivos ( $3^{a}$ amostragem), na camada de $0-5 \mathrm{~cm}$, permite constatar maior el evação dessa variável no tratamento em que o cal cário não foi incorporado, com val or significativamente superior ao SPC (Quadro 3). Essefato está deacordo com o resultado obtido por Prochnow et al. (2000), que verificaram maior el evação de pH na camada de 0-5 cm no SPD, 16 meses após a calagem. Portanto, no presente trabalho, o cal cário apresentou uma movimentação não só na camada superficial, mas também em profundidade no perfil do solo, pois os valores de $\mathrm{pH}$ de 5-10, 10-20 e 20-40 cm não diferiram significativamente dos apresentados no SPC. I sso implica dizer que, apesar de grande parte do calcário aplicado superficialmente, após 12 meses, continuar reagindo em superfície, parte substancial dos compostos da solubilização do corretivo acaba sofrendo uma movimentação vertical, atuando em profundidade.

No quadro 3, na $3^{3}$ amostragem e na profundidade de 0-5 cm, verifica-se que a aplicação da maior dose de calcário, independentemente da granulometria e do sistema de plantio, proporcionou el evação do pH em relação à menor dose. No entanto, a dose intermediária, estatisticamente, não diferiu desse resultado. Esse fato não se repetiu nas demais profundidades. Assim, mediante as constatações anteriores, questiona-se a redução da aplicação das doses de calcário em SPD, conforme sugere Wiethölter (2000), baseado nos estudos de Pöttker $\&$ Ben (1998).
Analisando, de forma conjunta, as três amostragens para pH (Quadro 3), constata-se que o material corretivo, independentemente do tratamento, continuou reagindo no solo até os 12 meses, sendo justamente na última amostragem que o $\mathrm{pH}$, na camada superficial $(0-5 \mathrm{~cm})$, atingiu valores máximos, próximos do ideal para o pleno desenvolvimento da maioria das culturas anuais. Para fins práticos, considera-se a faixa de $\mathrm{pH}$ entre 6,0 e 6,5 adequada para a maioria das plantas cultivadas no Brasil (POTAFOS, 1998), visto que, nessa faixa, aumenta a disponibilidade para os macronutrientes e afasta do mínimo ou da toxidez para os micronutrientes.

Semel hantemente ao que ocorreu com a acidez ativa do solo $(\mathrm{pH})$, os valores da acidez potencial $\left(\mathrm{H}+\mathrm{Al}\right.$ ), na $1^{\mathrm{a}}$ amostragem (Quadro 3), de forma geral, não diferiram entre os tratamentos. Como exceção, tem-se a profundidade de $5-10 \mathrm{~cm}$, no que diz respeito ao sistema de plantio, onde o SPC proporcionou maior decréscimo nos valores de $\mathrm{H}+\mathrm{Al}$. Como essa situação não se repetiu para nenhuma outra profundidade, torna-se difícil um entendimento para esse fato isolado.

$\mathrm{Na} 2^{\mathrm{a}}$ amostragem (Quadro 3), verificou-se o mesmo fato ocorrido com o pH, ou seja, não existiram diferenças significativas com relaçãoà granul ometria e doses. Já com relação ao sistema de plantio, observou-se que, nas camadas inferiores a $5 \mathrm{~cm}$, a incorporação do corretivo proporcionou maior eficiência na diminuição do $\mathrm{H}+\mathrm{Al}$, a exemplo do inverso ocorrido com o pH. Para a camada de 0$5 \mathrm{~cm}$, a aplicação do calcário, independentemente de granulometria e da dose, conferiu ao SPD o mesmo poder de neutralização do H + Al que oSPC. É possível quea quantidadeaplicada superficialmente tenha compensado a menor reação no solo, em relação ao SPC, onde o cal cário foi incorporado. O comportamento da acidez potencial, até então, foi o esperado, pois seguiu padrão inverso ao pH.

Doze meses após a aplicação do corretivo (Quadro 3), observou-se queda dos valores de H + Al, mostrando, mais uma vez, que o cal cário continuou a reagir no solo após três meses. Não houve diferenças significativas para granulometria edoses, porém verificou-se efeito com relação ao sistema de plantio. Na camada de $0-5 \mathrm{~cm}$, a concentração de calcário na superfície em SPD gerou uma contínua redução do $\mathrm{H}+\mathrm{Al}$ que, nesta fase de amostragem, superou os resultados doSPC. J á na camada de 10$20 \mathrm{~cm}$, um melhor controle da acidez potencial foi verificado, em função do SPC, justamente pela mobilização mecânica a que o sol o e o corretivo foram submetidos nessa profundidade.

$\mathrm{Na} 1^{\mathrm{a}}$ amostragem (Quadro 3), com relação aos níveis de $\mathrm{Ca}$, houve interação entre granulometria $x$ doses, na profundidade de $5-10 \mathrm{~cm}$, sendo significativos para Tukey a $5 \%$. Considerando o desdobramento da interação, verificou-se que, na 
dose 1/3, o calcário fino foi mais eficiente em el evar os teores de Ca no solo, sendo necessária a dosecompleta (3/3) do calcário grosso para que o mesmo tivesse atuação similar ao corretivo mais fino (Quadro 3).

Três meses após a aplicação do cal cário(Quadro 3), não se constataram diferenças entre tratamentos, a não ser para osistema de plantio, nas profundidades de $5-10,10-20$ e $20-40 \mathrm{~cm}$. Nas profundidades superiores a $5 \mathrm{~cm}$, os teores de Ca do tratamento SPD foram menores do que no SPC, mostrando que no SPD praticamente não houve movimentação de $\mathrm{Ca}$ em profundidade. Esse fato pode ser explicado pelo menor contato do calcário com o solo, acarretando hidrólise mais lenta. Além disso, para o corretivo reagir com os ácidos do solo, quesesituam apenas a frações de milímetros afastadas da superfície das partículas, os íons $\mathrm{Ca}^{2+} \mathrm{eOH}^{-}$têm de se movimentar até onde os ácidos se encontram, o que se dá predominantemente por difusão através da solução do solo. Dessa forma, com a saída dos íons $\mathrm{Ca}^{2+}$ e $\mathrm{OH}^{-}$da solução junto à superfície das partículas de cal cário, diminui ali sua concentração, permitindo que mais calcário se hidrolise. J á na camada de 0-5 cm, a menor quantidade de cal cário em superfície no SPC fez com que houvesse uma equivalência nos teores de $\mathrm{Ca}$ com os do SPD. Invariavelmente, no SPD, ocorre maior deposição de Ca na camada de $0-5 \mathrm{~cm}$, isso faz com que os resultados superem ou ao menos não se diferenciem quando comparados ao SPC.

A camada de 0-5 cm, após 12 meses da aplicação do corretivo (Quadro 3), apresentou teor de $\mathrm{Ca}$ superior no SPD do que no SPC. Na camada de 5-10 cm, tanto o SPD quanto o SPC apresentaram os mesmos val ores de Ca, indicando queos compostos liberados pela hidrólise do cal cário movimentaramse verticalmente no SPD. Esse resultado corrobora os obtidos por Caires et al. (1998) e Pöttker \& Bem (1998). Observa-se que, na camada de $10-20 \mathrm{~cm}$, houve inversão da situação, isto é, os teores de Ca no SPC superaram os do SPD. Entretanto, com o passar do tempo, o Ca poderá ser transportado para 15-20 cm de profundidade em SPD (Pavan, 1999).

Os tratamentos envolvendo granul ometria, após 12 meses da cal agem (Quadro 3), não apresentaram efeito significativo nas diferentes profundidades. Nesse período, a dose total de calcário mostrou-se superior na liberação de Ca, na camada de 0-5 cm. É provável que, atéà chegada desse período, ofator doses comece a tornar-se significativo, pela prol ongada atividade de reação do cal cário. Houve, também, interação entre granulometria $\mathrm{x}$ doses, na profundidade de $10-20 \mathrm{~cm}$ (Quadro 5). Verificou-se o inverso do quadro 4, só que para a dose de $2 / 3$, quando o calcário grosso proporcionou melhor desempenho no fornecimento de $\mathrm{Ca}$ ao solo, (Quadro 5). Esse fato pode ser explicado pel o efeito residual prolongado, ou seja, a maior parte da fração granulométrica mais fina (menor que $0,30 \mathrm{~mm}$ ) reage no solo em três meses, enquanto no calcário mais grosso ainda existe um resíduo com maior dimensão (2,0-0,30 mm), quecontinuará agindo mais lentamente após o período de três meses.

Os resultados da $1^{\text {a }}$ amostragem não diferiram para os teores de $\mathrm{Mg}^{2+}$ (Quadro 6), tendo sido observadas, apenas na última profundidade, diferenças com relação ao sistema de plantio e a granulometria. Entretanto, devem-seatribuir esses resultados mais às condições originais do solo (Quadro 1) do que propriamente aos efeitos de tratamentos. A $2^{a}$ amostragem, a exemplo da primeira, também não revelou diferenças entre os tratamentos. Somente aos 12 meses da aplicação dos corretivos, observou-se significância para o sistema de plantio e doses. Nesse período, o comportamento do $\mathrm{M} \mathrm{g}^{2+}$, com relação ao sistema de plantio, foi idêntico ao apresentado pelo $\mathrm{Ca}^{2+}$ até à profundidade de $20 \mathrm{~cm}$, cabendo o mesmo raciocínio para explicação. Na primeira camada, os teores de $\mathrm{Mg}^{2+}$ do SPD foram superiores aos do SPC e, na profundidade imediatamente inferior, verificou-se equivalência entreos SP, mostrando a movimentação do $\mathrm{Mg}$, mesmo quando o calcário foi aplicado superficialmente. J á a partir da camada de $10-20 \mathrm{~cm}$, o SPC passou a apresentar maiores teores de $\mathrm{Mg}^{2+}$.

\section{Quadro 4. Teores de cálcio do solo, considerando a granulometria e doses de calcários, após a $1^{\mathrm{a}}$ amostragem, na profundidade de $5-10 \mathrm{~cm}$. (Desdobramento da interação)}

\begin{tabular}{cccc}
\hline \multirow{2}{*}{ Granulometria } & \multicolumn{3}{c}{ Dose de calcário } \\
\cline { 2 - 4 } & $\mathbf{1 / 3}$ & $\mathbf{2 / 3}$ & $\mathbf{3 / 3}$ \\
\hline & \multicolumn{3}{c}{$\mathrm{mmol}_{\mathrm{c}} \mathrm{dm}^{-3}$} \\
Grosso & $13 \mathrm{bA}$ & $13 \mathrm{aA}$ & $21 \mathrm{aA}$ \\
Fino & $21 \mathrm{aA}$ & $12 \mathrm{aA}$ & $15 \mathrm{aA}$
\end{tabular}

Médias seguidas das mesmas letras (minúsculas, na coluna, e maiúsculas, na linha) não diferem entresi peloteste de Tukey a $5 \%$

Quadro 5. Teores de cálcio do solo, considerando a granulometria e doses de calcários, após a $3^{\mathrm{a}}$ amostragem, na profundidade de $10-20 \mathrm{~cm}$. (Desdobramento da interação)

\begin{tabular}{cccc}
\hline \multirow{2}{*}{ Granulometria } & \multicolumn{3}{c}{ Dose de calcário } \\
\cline { 2 - 4 } & $\mathbf{1 / 3}$ & $\mathbf{2 / 3}$ & $\mathbf{3 / 3}$ \\
\cline { 2 - 4 } & $\mathrm{mmol}_{\mathrm{c}} \mathrm{dm}^{-3}$ & \\
Grosso & $17 \mathrm{aA}$ & $27 \mathrm{aA}$ & $21 \mathrm{aA}$ \\
Fino & $20 \mathrm{aA}$ & $16 \mathrm{bA}$ & $24 \mathrm{aA}$
\end{tabular}

Médias seguidas das mesmas letras (minúsculas, na coluna, e maiúsculas, na linha) não diferem entresi pelo teste de Tukey a $5 \%$. 
Quadro 6. Teores de magnésio trocável do solo em diferentes profundidades, após 1, 3 e 12 meses da aplicação do corretivo, considerando o sistema de plantio (SP), a granulometria (Gran) e as doses de calcário (Doses)

\begin{tabular}{|c|c|c|c|c|c|c|c|c|c|c|c|c|}
\hline \multirow{2}{*}{ Fator } & \multicolumn{4}{|c|}{ 1a amostragem (1 mês) - Prof. (cm) } & \multicolumn{4}{|c|}{$2^{\mathrm{a}}$ amostragem (3 meses) - Prof. (cm) } & \multicolumn{4}{|c|}{$3^{\mathrm{a}}$ amostragem (12 meses) - Prof. (cm) } \\
\hline & $0-5$ & $5-10$ & $10-20$ & $20-40$ & $0-5$ & 5-10 & $10-20$ & $20-40$ & $0-5$ & 5-10 & $10-20$ & $20-40$ \\
\hline \multicolumn{13}{|c|}{$\mathrm{Mg}^{2+}, \mathbf{m m o l}_{\mathbf{c}} \mathbf{d m}^{-3}$} \\
\hline \multicolumn{13}{|c|}{ Sistema de plantio } \\
\hline Convencional & 11 a & $10 \mathrm{a}$ & $10 \mathrm{a}$ & $7 \mathrm{a}$ & $13 \mathrm{a}$ & $13 \mathrm{a}$ & $15 \mathrm{a}$ & $12 \mathrm{a}$ & $23 b$ & 19 a & 15 a & $6 a$ \\
\hline Direto & $10 a$ & 9 a & 7 a & $5 b$ & 14 a & $12 \mathrm{a}$ & 13 a & 11 a & 38 a & $21 \mathrm{a}$ & $10 \mathrm{~b}$ & $5 b$ \\
\hline \multicolumn{13}{|c|}{ Granulometria } \\
\hline Grosso & 9 a & 8 a & 8 a & 7 a & 12 a & 12 a & $15 \mathrm{a}$ & 12 a & 30 a & 19 a & 13 a & 5 a \\
\hline Fino & $11 \mathrm{a}$ & $10 \mathrm{a}$ & $8 a$ & $6 \mathrm{~b}$ & 15 a & 13 a & 13 a & $12 \mathrm{a}$ & 31 a & 21 a & 13 a & 5 a \\
\hline \multicolumn{13}{|c|}{ Doses } \\
\hline $1 / 3$ & 8 a & 7 a & $8 a$ & $6 a$ & 17 a & $14 \mathrm{a}$ & $13 a$ & $12 \mathrm{a}$ & $21 b$ & 19 a & 12 a & $5 a$ \\
\hline $2 / 3$ & 13 a & $12 \mathrm{a}$ & $10 \mathrm{a}$ & $7 \mathrm{a}$ & 12 a & $10 \mathrm{a}$ & $14 \mathrm{a}$ & $11 \mathrm{a}$ & $29 b$ & 18 a & $13 a$ & $5 a$ \\
\hline $3 / 3$ & $10 \mathrm{a}$ & 9 a & $8 a$ & 6 a & 12 a & $13 a$ & 15 a & 13 a & 42 a & $24 a$ & 13 a & $6 a$ \\
\hline SP & 0,06 & 0,38 & 2,65 & $2,14 *$ & 0,02 & 0,63 & 0,62 & 0,51 & $16,13 *$ & 0,20 & $3,49 *$ & $2,51^{*}$ \\
\hline Gran & 1,04 & 4,01 & 2,30 & $1,98 *$ & 4,44 & 0,86 & 2,39 & 0,08 & 0,24 & 1,49 & 0,01 & 0,01 \\
\hline Dose & 1,92 & 4,14 & 1,08 & 1,32 & 0,99 & 1,21 & 0,26 & 1,14 & $11,98 *$ & 2,07 & 0,80 & 2,85 \\
\hline SP x Gran & 0,04 & 0,58 & 0,69 & 0,08 & 3,07 & 0,86 & $10,07 *$ & 2,07 & 2,75 & 3,81 & 2,55 & 3,78 \\
\hline SP $\times$ Dose & 0,30 & 0,06 & 1,27 & 2,26 & 0,54 & 2,68 & 0,55 & 0,38 & 2,17 & 0,59 & 0,37 & 0,17 \\
\hline Gran x Dose & 0,15 & 0,57 & 1,55 & 0,16 & 1,75 & 2,94 & 0,55 & 1,26 & 0,18 & 2,19 & $4,91^{*}$ & 0,17 \\
\hline \multicolumn{13}{|c|}{ C.V. (\%) } \\
\hline SP & 25 & 24 & 32 & 27 & 24 & 21 & 21 & 19 & 18 & 21 & 34 & 27 \\
\hline Gran & 39 & 25 & 27 & 33 & 24 & 37 & 15 & 22 & 9 & 23 & 27 & 50 \\
\hline Dose & 63 & 51 & 44 & 38 & 79 & 55 & 60 & 36 & 42 & 42 & 36 & 39 \\
\hline
\end{tabular}

Médias seguidas por letras iguais, nas colunas, não diferem entre si pelo teste de Tukey a 5 \%. * significativo a 5 \% pel o teste $\mathrm{F}$.

Os resultados referentes à $3^{a}$ amostragem (Quadro 6), evidenciam quea maior dose decal cário proporcionou elevação dos teores de $\mathrm{Mg}^{2+}$ na camada de $0-5 \mathrm{~cm}$; tal fato não se repetiu nas demais profundidades. Houve a interação granulometria x doses, significativa no teste de comparação de médias, na profundidade de $10-20 \mathrm{~cm}$ (Quadro 7). Essa interação revela diferenças de granulometria na primeira dose, com aumento do teor de $\mathrm{Mg}^{2+}$ no cal cário fino, e, na dose cheia, o $\mathrm{Mg}^{2+}$ foi igual tanto para o cal cário fino como para o grosso.

Quadro 7. Teores de magnésio trocável do solo, considerando a granulometria e doses de calcário, após a $3^{\mathrm{a}}$ amostragem, na profundidade de $10-20 \mathrm{~cm}$. (Desdobramento da interação)

\begin{tabular}{crcc}
\hline \multirow{2}{*}{ Granulometria } & \multicolumn{3}{c}{ Dose de calcário } \\
\cline { 2 - 4 } & $\mathbf{1 / 3}$ & $\mathbf{2 / 3}$ & $\mathbf{3 / 3}$ \\
\hline & & $\mathrm{mmol}_{\mathrm{c}} \mathrm{dm}^{-3}$ & \\
& & $16 \mathrm{aA}$ & $13 \mathrm{aAB}$ \\
Grosso & $9 \mathrm{aB}$ & $10 \mathrm{bA}$ & $14 \mathrm{aA}$ \\
Fino & $14 \mathrm{aA}$ & &
\end{tabular}

Médias seguidas das mesmas letras (minúsculas, na coluna, e maiúsculas, na linha) não diferem entresi pelo teste deTukey a $5 \%$.
Os resultados obti dos nestetrabal ho, com relação à redução da acidez de superfície e de subsuperficie, mostram ser possível realizar a correção da acidez do solo por meio da calagem superficial na fase de instalação doSPD. Diversos mecanismos, esuas ações conjuntas, podem ser responsáveis em levar os produtos da dissolução do calcário a atuarem em profundidade, como: o deslocamento de finas partículas decal cário através da porosidade contínua no perfil do solo, a presença de canalículos formados por raízes mortas egalerias da mesofauna, a formação de pares de $\mathrm{Ca}^{2+} \mathrm{eMg}^{2+} \mathrm{com} \mathrm{NO}_{3}{ }^{-} \mathrm{eSO}_{4}{ }^{-2}$ defertilizantes ou liberados pela mineralização da M.O. ou, ainda, por ligantes orgânicos produzidos na decomposição dos resíduos vegetais (Petrere \& Anghinoni, 2001).

\section{CONCLUSÕES}

1. A aplicação de calcário em superfície na instalação dosistema plantio direto, independentementeda granulometria e da dose do material corretivo, alterou positivamente os atributos químicos do solo

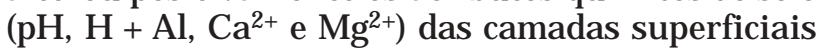
(0-5 e 5-10 cm), 12 meses após a calagem.

2. A aplicação de doses mais el evadas de cal cário, com maior granulometria, mostrou efeito residual prolongado para a correção da acidez do solo. 


\section{AGRADE CIMENTOS}

À Empresa Brasileira de Calcário Ltda. (EMBRACAL), por meio de sua associada Calcário Bonança, pel os corretivos especialmente preparados para o experimento.

\section{LITE RATURA CITADA}

ALCARDE, J.C.; PAULINO, V.T. \& DERNARDIN, J.S. Avaliação da reatividade de corretivos da acidez do sol o. $R$. Bras. Ci. Solo, 13:387-392, 1989.

ASSOCIAÇÃO BRASILEIRA PARA PESQUISA DA POTASSA E DO FOSFATO - POTAFOS. Manual internacional de fertilidade do solo. 2.ed. Piracicaba, 1998. 177p.

CAIRES, E.F.; CHUEIRI, W.A.; MADRUGA, E.F. \& FIGUEIREDO, A. Alterações de características químicas do solo e resposta da soja ao calcário e gesso aplicados na superfície em sistema de cultivo sem preparo do solo. R. Bras. Ci. Solo, 22:27-34, 1998.

CAIRES, E.F.; FONSECA, A.F.; MENDES, J .; CHUEIRI, W.A. \& MADRUGA, E.F. Produção de milho, trigo e soja em função das alterações das características químicas do solo pela aplicação de cal cário e gesso na superfície, em sistema de plantio direto. R. Bras. Ci. Solo, 23:315-327, 1999.

CAIRES, E.F.; BLUM, J . \& FELDHAUS, I.C. Resposta da soja ao calcário e gesso aplicados na implantação do sistema plantio direto. In: FERTIBIO, Santa Maria, 2000. Resumos Expandidos. Santa Maria, Universidade Federal de Santa Maria, 2000. CD-ROM

CHITOLINA, J.C.; PRATA, F.; SILVA, F.C.; MURAOKA, T. \& VITTI, A.C. Amostragem, acondicionamento e preparo de amostras de solo para análise de fertilidade. In: SILVA, F.C., ed. Manual de análises químicas de solos, plantas e fertilizantes. Brasília, EMBRAPA Comunicação para Transferência de Tecnologia, 1999. p.11-48.

DELAVALE, F.G.; LAZARINI, E. \& BUZETTI, S. Efeitos de cobertura e manejo do cal cário na implantação do sistema plantio direto em solo característico de cerrado. In: FERTIBIO, Santa Maria, 2000. Resumos Expandidos. Santa Maria, Universidade Federal de Santa Maria, 2000. CD-ROM

DERPSCH, R. \& FLORENTIN, M. Direktsaat nachaltige landwirtschaf ohne bodenbearbeitung, entwicklung + ländlicher raum. DLG-Verlags-GmbH, 34:22-25, 2000.

EMPRESA BRASILEIRA DE PESQUISA AGROPECUÁRIA EMBRAPA. CentroNacional de Pesquisa de Solos. Sistema Brasileiro de Classificação de Solos. Rio de J aneiro, 1999. 412p. (EMBRAPA-Solos. Documentos, 15)

LABORATÓRIO NACIONAL DE REFERÊNCIA VEGETAL LANARV. Análise de corretivos, fertilizantes einoculantes: métodos oficiais. Brasília, 1988. 104p.

LIMA, E.V.\& CRUSCIOL, C.A.C. Fertilidade do sol o no sistema de semeadura direta recém implantado em função da cobertura vegetal e da calagem superficial. In: CONGRESSO BRASILEIRO DE CIÊNCIA DO SOLO, Londrina, 2001. Resumo. Londrina, Sociedade Brasileira de Ciência do Solo, 2001. p.130.
PAVAN, M.A. Mobilização orgânica do calcário no solo através de adubo verde. In: PAULETTI, V. \& SEGANFREDO, R., eds. Plantio direto: atualização tecnológica. São Paulo, Fundação Cargill/F undação ABC, 1999. p.45-52.

PETRERE, C. \& ANGHINONI, I. Alteração de atributos químicos no perfil do solo pela calagem superficial em campo nativo. R. Bras. Ci. Solo, 25:885-895, 2001.

PÖTTKER, D. \& BEN,J .R. Calagem para uma rotação deculturas no sistema plantio direto. R. Bras. Ci. Solo, 22:675-684, 1998.

PROCHNOW, D.; ELTZ, F.L.F.; WENDLING, A.; STRECK, C.A. \& WENDLING, B. Alternativas de calagem em sistema de plantio direto. In: FERTIBIO, Santa Maria, 2000. Anais. Santa Maria, Universidade Federal de Santa Maria, 2000. CD-ROM

QUAGGIO,J .A. Acidez e calagem em sol os tropicais. Campinas, Instituto Agronômico, 2000. 111p.

RAIJ , B. van; CANTARELLA, H.; QUAGGIO, J .A. \& FURLANI, A.M.C. Recomendações de calagem e adubação para o estado de São Paulo. Campinas, Instituto Agronômico de Campinas, 1997. 285p. (Boletim Técnico, 100)

RAIJ , B. van \& CANTARELLA, H. Cereais: milho para grão e silagem. In: RAIJ , B. van; CANTARELLA, H.; QUAGGIO, J .A. \& FURLANI, A.M.C., eds. Recomendações de calagem eadubação para o estado de São Paulo. Campinas, Instituto Agronômico de Campinas, p.56-59, 1997. (Boletim técnico, 100)

RAIJ , B. van \& QUAGGIO, A.J. Métodos de análise de solo para fins de fertilidade. Campinas, Instituto Agronômico de Campinas, 1983. 31p. (Boletim técnico, 81)

RHEINHEIMER, D.S.; SANTOS, E.J .S.; KAMINSKI, J .; BORTOLUZZI, E.C. \& GATIBONI, L.C. Alterações deatributos do solo pela calagem superficial e incorporada a partir de pastagem natural. R. Bras. Ci. Solo, 24:797-805, 2000.

SÁ, J .C.M. Calagem em solos sob plantio direto da região dos campos gerais, centro-sul do Paraná. In: CURSO SOBRE MANEJ O DO SOLO NO SISTEMA PLANTIO DIRETO, Castro, 1995. Anais. Castro, Fundação ABC, 1995. p.73-107.

SALET, R.L. Dinâmica de íons na solução de um sol o submetido ao sistema plantio direto. Porto Alegre, Universidade Federal do RioGrandedoSul, 1994. 110p. (TesedeMestrado)

SOUZA, D.M.G. Manejo da fertilidade do sol o no sistema plantio direto: experiência no cerrado. In: FERTIBIO, Santa Maria, 2000. Anais. Santa Maria, Universidade Federal de Santa Maria, 2000. CD-ROM

TEDESCO, M.J . \& GIANELLO, C. Escol ha do corretivo da acidez do solo. In: CORRETIVOS DA ACIDEZDO SOLO, 2., Santa Maria, 1989. Anais. Santa Maria, Universidade Federal de Santa Maria, 1989. p.108-129.

WIETHÖLTER, S. Manejo da fertilidade do solo no sistema plantio direto: experiência nos estados do Rio Grande do Sul e Santa Catarina. In: FERTIBIO, Santa Maria, 2000. Anais. Santa Maria, UniversidadeF ederal deSanta Maria, 2000. CD-ROM 